\title{
SOME INEQUALITIES FOR UNITARILY INVARIANT NORMS
}

\author{
JUNLIANG WU AND JiANGUO ZHAO
}

Abstract. In this note, we use the convexity of the function $\varphi(v)$ to sharpen the matrix version of the Heinz means, where $\varphi(v)$ is defined as $\varphi(v)=\left\|A^{v} X B^{1-v}+A^{1-v} X B^{v}\right\|$ on $[0,1]$ for $A, B, X \in M_{n}$ such that $A$ and $B$ are positive semidefinite, and also give a refinement of the inequality [Theorem 6, SIAM J. Matrix Anal. Appl. 20 (1998), 466-470] which is due to Zhan.

Mathematics subject classification (2010): 15A60, 47A30.

Keywords and phrases: Heinz means, unitarily invariant norm, positive semidefinite matrix.

\section{REFERENCES}

[1] R. Bhatia, Matrix Analysis, Springer, New York, 1997.

[2] R. BHATIA AND C. DAVIS, More matrix forms of the arithmetic-geometric mean inequality, SIAM J. matrix Anal. Appl. 14, (1993), 132-136.

[3] R. Bhatia, Positive Definite Matrices, Princeton University Press, New Jersey, 2007.

[4] R. Bhatia And F. Kittaneh, Notes on matrix arithmetic-geometric mean inequalities, Linear Algebra Appl. 308, (2000), 203-211.

[5] C. Conde, M. S. Moslehian And A. Seddik, Operator inequalities related to the Corach-PortaRecht inequality, Linear Algebra Appl. 436, (2012), 3008-3017.

[6] G. Corach, R. Porta And L. Recht, An operator inequality, Linear Algebra Appl. 142, (1990), $153-158$.

[7] I. C. Gohberg AND M. G. KREIN, Introduction to the theory of Linear Non-self-adjoint operators, Transl. Math. Monographs, vol. 18, AMS, Providence, RI, 1969.

[8] F. HIAI AND H. KosAKI, Means for matrices and comparison of their norms, Indiana Univ. Math, J. 48, (1999), 899-936.

[9] F. Hiai And H. KosaKi, Means of Hilbert space operators, Lecture Notes in Mathematics, vol. 1820, Springer, New York, 2003.

[10] R. A. Horn And C. R. Johnson, Topics in Matrix Analysis, Cambridge University Press, New York, 1991.

[11] F. KitTaneh, On the convexity of the Heinz means, Inter. Equ. Oper. Theory 68, (2010), 519-527.

[12] J. S. Matharu AND J. S. Aujla, Some inequalities for unitarily invariant norms, Linear Algebra Appl. 436, (2012), 1623-1631.

[13] J. E. Pecaric And P. R. Bees ACK, On Jessen's Inequality for Convex Functions II, J. Math. Anal. Appl. 118, (1986), 125-144.

[14] R. Schatten, Norm Ideals of Completely Continuous Operators, Springer, Berlin, 1960.

[15] B. Simon, Trace Ideals and Their Applications, Cambridge University Press, Cambridge, 1979.

[16] X. ZHAN, Inequalities for unitarily invariant norms, SIAM J. Matrix Anal. Appl. 20, (1998), 466-470.

[17] L. M. ZOU AND C. J. HE, Weak majorization inequalities for singular values, Operators and Matrices, 7 (3), (2013), 733-437. 